\title{
CONNECTIVITY OF THE GRAPHS OF SEMIRINGS: LIFTING AND PRODUCT
}

\author{
Y.-F. LIN AND J. S. RATTI
}

1. Introduction. A semiring is a nonempty set $R$ equipped with two binary operations, called addition + and multiplication (denoted by juxtaposition), such that $R$ is multiplicatively a semigroup and additively a commutative semigroup, and that the multiplication is distributive across the addition both from the left and from the right. Contrary to [1], the existence of a zero element in a semiring is not assumed.

By the $\operatorname{graph} G(R)$ of a semiring $R$, we mean the nonoriented graph whose vertices consists of the set $R$ of all proper subsemirings of $R$ in which two vertices $V_{1}$ and $V_{2}$ are adjacent, or joined by an edge, if and only if $V_{1} \cap V_{2} \neq \square$, where $\square$ denotes the empty set. The graph $G(R)$ is said to be connected if and only if for each pair or vertices $V$ and $V^{\prime}$ there exists a finite sequence, called a path, of vertices $V$ $=V_{1}, V_{2}, \cdots, V_{n+1}=V^{\prime}$ such that every two consecutive vertices are adjacent. It is conjectured [4] that the graph of a semiring with more than two elements is connected. ${ }^{1}$ Similar problems have been considered by Bosák [2], Lin [3], Ponděliček [5], and the authors [4].

The purpose of this paper is to prove the following results. As an application of Theorem 1, we show that the graphs of free semirings are all connected.

TheOREM 1 (Lifting). If a semiring $R$ has a homomorphic image $R^{\prime}$ whose graph is nonempty and connected, then the graph of $R$ is connected.

TheOREM 2 (PRODUCT). If $\left\{R_{\lambda} \mid \lambda \in \Lambda\right\}$ is a family of semirings that contains a semiring whose graph is nonempty and connected, then the Cartesian product $\Pi_{\lambda} R_{\lambda}$ has a connected graph.

Examples are given to show that the converses of Theorems 1 and 2 are both not true.

2. Definitions and preliminaries. If $R_{1}$ and $R_{2}$ are two semirings, we shall write $R_{1} \times R_{2}$ for the semiring under the operations:

Received by the editors June 16, 1969.

${ }^{1}$ Example of semiring with two elements that has a disconnected graph exists. See Example 1. 


$$
\begin{aligned}
(a, b)+(c, d) & =(a+c, b+d), \\
(a, b)(c, d) & =(a c, b d)
\end{aligned}
$$

for all $(a, b)$ and $(c, d)$ in the Cartesian product $R_{1} \times R_{2}$. Similarly, the Cartesian product $\Pi_{\lambda} R_{\lambda}$ of any family $\left\{R_{\lambda}\right\}$ of semirings is a semiring. A homomorphism from a semiring $R$ to a semiring $R^{\prime}$ is a function $h: R \rightarrow R^{\prime}$ such that $h(a+b)=h(a)+h(b)$ and $h(a b)=h(a) h(b)$ for all $a$ and $b$ in $R$. For any $x_{1}, x_{2}, \cdots, x_{n}$ in $R$, we shall write $\left\langle x_{1}, x_{2}\right.$, $\left.\cdots, x_{n}\right\rangle$ for the subsemiring of $R$ generated by $\left\{x_{1}, x_{2}, \cdots, x_{n}\right\}$. Thus, $\left\langle x_{1}, x_{2}, \cdots, x_{n}\right\rangle$ is the intersection of all the subsemirings of $R$ containing $\left\{x_{1}, x_{2}, \cdots, x_{n}\right\}$.

The trivial semiring, which consists of a single element alone, has an empty graph. We shall call a semiring nontrivial if it contains more than one element.

The only known example of a semiring with a disconnected graph is the following

EXAMPLE 1 . Let $R_{2}$ be the set $\{0,1\}$ together with the addition and the multiplication given by the following tables:

\begin{tabular}{l|lll|ll}
+ & 0 & 1 & $\cdot$ & 0 & 1 \\
\hline 0 & 0 & 1 & 0 & 0 & 0 \\
1 & 1 & 1 & 1 & 0 & 1
\end{tabular}

$R_{2}$ is a semiring with exactly two proper subsemirings $\{0\}$ and $\{1\}$ and hence its graph, which consists of two isolated vertices, is not connected.

3. Proofs of main results. For our later convenience, we first establish the following useful

LEMmA. A semiring $R$ is nontrivial if and only if there exists an element $x \in R$ such that $\langle x\rangle \neq R$.

Proof. Only the "only if" part needs a proof. We prove the contrapositive of this. Let $R$ be a semiring such that $\langle y\rangle=R$ for every $y \in R$. For any $x \in R$, since $R=\left\langle x^{2}\right\rangle$ and since members of $\left\langle x^{2}\right\rangle$ are those polynomials in $x^{2}$ with natural numbers as coefficients (e.g. $2 x^{2}=x^{2}+x^{2}$ ) and without any constant term, we have,

$$
x=n_{1} x^{2 k_{1}}+n_{2} x^{2 k_{2}}+\cdots+n_{p} x^{2 k_{p}}
$$

for some natural numbers $n_{1}, \cdots, n_{p} ; k_{1}, \cdots, k_{p}$. Denote $e=n_{j} x^{2 k_{1}-1}+n_{2} x^{2 k_{2}-1}+\cdots+n_{p} x^{2 k_{p}-1}$. Then $x=e x$, which implies that 
$e$ is multiplicatively a left unit (=left identity) for $\langle x\rangle=R$. Since by assumption

$$
\begin{aligned}
R & =\langle e\rangle=\{e, 2 e, 3 e, \cdots\} \\
& =\langle 2 e\rangle=\{2 e, 4 e, 6 e, \cdots\}
\end{aligned}
$$

we must have

$$
e=2 m e \text { for some integer } m \geqq 1 \text {. }
$$

Let $z=(2 m-1) e$. Then $e=z+e$, and $z e=(2 m-1) e e=(2 m-1) e=z$. Similarly $e z=z$. Thus, $z$ is additively and multiplicatively the zero element for $\langle e\rangle=R$. Consequently, the semiring $\langle z\rangle=\{z\}=R$ is trivial.

Corollary. A semiring has a nonempty graph if and only if it is nontrivial.

Proof. Straightforward.

Proof For Theorem 1. Let $h: R \rightarrow R^{\prime}$ be a homomorphism of $R$ onto $R^{\prime}$, and let $V$ and $W$ be any two proper subsemirings of $R$. We shall exhibit a path in $G(R)$ connecting $V$ and $W$ : Since $h$ is a homomorphism, $h(V)$ and $h(W)$ are two (not necessarily proper) subsemirings of $R^{\prime}$. By virtue of the lemma we have just proved, if $h(V)=R^{\prime}=h(W)$ then there exist $v \in V$ and $w \in W$ such that $\langle h(v)\rangle$ and $\langle h(w)\rangle$ are two proper subsemirings of $R^{\prime}$. The last conclusion is obviously true when $h(V) \neq R^{\prime}$ and/or $h(W) \neq R^{\prime}$. The connectedness of the graph of $R^{\prime}$ implies that there is a path

$$
\langle h(v)\rangle, V_{1}^{\prime}, V_{2}^{\prime}, \cdots, V_{n}^{\prime},\langle h(w)\rangle
$$

in $G\left(\mathcal{R}^{\prime}\right)$ connecting $\langle h(v)\rangle$ and $\langle h(w)\rangle$. It follows that

$$
V, h^{-1}(\langle h(v)\rangle), h^{-1}\left(V_{1}^{\prime}\right), h^{-1}\left(V_{2}^{\prime}\right), \cdots, h^{-1}\left(V_{n}^{\prime}\right), h^{-1}(\langle h(v)\rangle), W
$$

forms a path in $G(\Omega)$ connecting $V$ and $W$. Hence, the graph of $R$ is connected.

Proof For Theorem 2 . Let $R_{\mu}$ denote a member of the family $\left\{R_{\lambda} \mid \lambda \in \Lambda\right\}$ such that the graph of $R_{\mu}$ is nonempty and connected, and let

$$
\pi_{\mu}: \prod_{\lambda \in \Lambda} R_{\lambda} \rightarrow R_{\mu}
$$

be the $\mu$ th projection. Then since $\pi_{\mu}$ is an epimorphism, by Theorem 1 the graph of $\Pi_{\lambda \in \Lambda} R_{\lambda}$ is connected.

Definition. By a free semiring generated by a set $S$ we mean a semiring $F \supset S$ such that for every function $g: S \rightarrow R$ from the set $S$ to 
a semiring $R$, there exists a unique homomorphism $h: F \rightarrow R$ satisfying the commutativity of the following diagram:

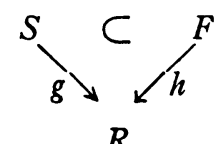

It is easy to see that for any given nonempty set $S$, a free semiring (generated by $S$ ) exists, and that all the free semirings generated by the same set are isomorphic to each other.

THEOREM 3. The graph of a free semiring is connected.

Proof. Let $F$ be a free semiring generated by a set $S$. Then $S$ is necessarily nonempty. Choose any $x \in S$, and consider the subsemiring $\langle x\rangle$ of $F$ generated by the single element $x$. Since $\langle x\rangle$ is a nontrivial commutative semiring, its graph is nonempty and connected [4]. Let $g: S \rightarrow\langle x\rangle$ be the function defined by $g(t)=x$ for all $t \in S$. Then since $F$ is the free semiring generated by $S$, there exists a homomorphism $h: F \rightarrow\langle x\rangle$ such that $h \mid S=g$. It follows that $h(F)=\langle x\rangle$ and hence, by Theorem 1, the graph of $F$ is connected.

4. Remarks. It is natural to ask whether the connectedness of the graphs of semirings is preserved by the epimorphisms, that is whether the converse of Theorem 1 is true. The following example shows that the answer is in the negative.

EXAmple 2. Let $R_{2}$ be the semiring of Example 1, and let $R=$ $R_{2} \times R_{2}$. A routine verification shows that the graph of $R$ is connected. Let $h: R \rightarrow R_{2}$ be the first (or second) projection of $R$ onto $R_{2}$. Then $h$ is an epimorphism. However, the graph of $h(R)=R_{2}$ is, as we have seen in Example 1, not connected.

Example 2 also shows the converse of Theorem 2 is not true.

\section{REFERENCES}

1. Paul J. Allen, A fundamental theorem of homomorphisms for semirings, Proc. Amer. Math. Soc. 21 (1969), 412-416.

2. J. Bosák, The graphs of semigroups, Theory of graphs and its applications, Proc. Sympos. (Smolenice, 1963) Publ. House Czechoslovak Acad. Sci., Prague, 1964, pp. 119-125. MR 30 \#3928.

3. Y.-F. Lin, A problem of Bosak concerning the graphs of semigroups, Proc. Amer. Math. Soc. 21 (1969), 343-346.

4. Y.-F. Lin and J. S. Ratti, The graphs of semirings, J. Algebra (to appear).

5. Bedřich Ponděliček, Diameter of a graph of a semigroup, Časopis Pěst. Mat. 92 (1967), 206-211. MR 36 \#6323.

University of South Florida 